\title{
Cochrane corner: patient safety in cataract surgery
}

\author{
Gerry Clare ${ }^{1}$
}

Received: 18 May 2019 / Accepted: 22 May 2019 / Published online: 9 July 2019

(c) The Royal College of Ophthalmologists 2019

The efficacy and cost of routine pre-operative screening for medical conditions before cataract surgery have been evaluated in a recent Cochrane Review [1]. The authors identified three randomised controlled trials (RCTs) [2-4] in which a total of 21,531 patients scheduled to have surgery under local anaesthesia for age-related cataract either underwent routine tests (including electrocardiography, full blood counts and various other serum tests), or had no testing other than specific tests like blood glucose levels in the case of diabetic patients. The results of the systematic review indicate that the risk of intra- and post-operative medical adverse events (mostly cardiovascular, such as hypertension requiring initiation of medical treatment) is $3.3 \%$ and is not reduced by routine pre-operative screening tests. Medical adverse events resulted in the cancellation of cataract surgery in $2 \%$ of cases in each group. The authors carried out a secondary analysis of ocular adverse events, finding no significant difference between the two categories in two of the three RCTs [2, 3] $(\sim 6.8 \%)$. The cost of cataract surgery was estimated in one study to be 2.55 times higher for those patients undergoing routine tests [3]. The overall risk of bias in the RCTs was assessed to be low.

By demonstrating that patient safety is not improved by routinely screening for medical conditions prior to cataract surgery, this analysis highlights the potential for efficiency savings by reducing unnecessary tests. While this is a robust effort to identify wastage, it is doubtful that many public sector service providers offer routine screening tests, suggesting that this may be more of a concern in a private setting. It is puzzling, given that hypertension is the most commonly encountered adverse event in the review, that the authors do not specifically mention the role of pre-operative

Gerry Clare

gc.island@gmail.com

1 Cochrane Eyes and Vision Group International Centre for Eye Health London School of Hygiene \& Tropical Medicine, London WC1E 7HT, UK blood pressure measurement in managing patient risk; perhaps this is considered to be a basic and universally mandatory pre-operative screening test, but this cannot be assumed.

Taking a broader view, patient safety concerns in cataract surgery are not restricted to adverse medical events, but also to operative complications and poor outcomes. It is well known, for example, that posterior capsule rupture during cataract surgery significantly increases the risk of endophthalmitis and retinal detachment [5]. Unsurprisingly, two RCTs in this analysis found the rates of clinically important ocular adverse events, the most common of which was posterior capsule rupture with and without vitreous loss, to be equal between both groups.

This prompts the question of how best to identify the general medical and ocular factors that lead to surgical complications, if not by routine medical testing. Increasingly, surgical providers are adopting formal risk assessment and stratification methods, rather than a clinical assessment alone, to identify and mitigate the specific risk factors associated with poor outcomes in cataract surgery [6]. Formal risk stratification is a systematic method of scoring known risk factors and may take the form of a traffic light system $(\mathrm{red}=$ high risk, amber $=$ intermediate risk, green $=$ low risk) or similar.

Known ocular factors making vitreous loss more likely include hyperdense and white crystalline lenses, phacodonesis, a small pupil and a shallow anterior chamber. Medical risk factors include obesity, poor mobility, cognitive impairment and extremes of age. Prospective studies have been carried out to evaluate the numerical probability of surgical complications associated with different risk categories, validating the stratification methods used [7]. These systems take into account both the likelihood and possible consequences of surgical complications. Thus, while a patient with only one seeing eye does not necessarily have a higher numerical risk of a surgical complication, the consequences are obviously more severe, and this can be factored in to the assessment. These methods of risk management may be most appropriate in teaching 
environments, where the assessors and surgeons may be relatively junior and may not fully appreciate the importance of certain clinical findings.

Crucially, appropriate risk stratification allows the assessor to plan additional measures such as using dispersive viscoelastic, trypan blue dye or iris hooks, or to request a senior surgeon to operate. Moreover, the concept of risk need not be limited to intra-operative complications but can be extended to include less favourable outcomes such as unsatisfactory post-operative refractive errors and cystoid macular oedema. The risk assessment pathway can therefore incorporate a precise surgical plan, including method of anaesthesia, intraocular lens choice, management of astigmatism and post-operative drop regimens. It is this ophthalmologist's experience that in the absence of a formal surgical plan, important clinical details-such as the presence of endothelial guttae, the position of the steep axis or a previous refractive surprise in the fellow eye-can be forgotten at the time of surgery and may detract from the quality of the outcome.

While it is conceivable that a formal risk assessment reduces the risk of complications and adverse outcomes, this has not been demonstrated by RCTs, and consequently is not universally practiced. Detractors of risk stratification are apt to view it as lengthy and superfluous. Future RCTs in this field are therefore needed to compare the effects of formal risk stratification versus clinical assessment alone on outcomes of cataract surgery. Since serious intra-operative complications are relatively rare [5], large numbers of eyes would be required to show statistically significant differences.

Many questions concerning cataract surgery techniques and outcomes remain unanswered and could be addressed by RCTs. For example, the role of primary manual small incision cataract surgery in the management of hyperdense nucleosclerotic cataracts remains incompletely defined. This technique might conceivably be safer than phacoemulsification in certain cases. A recent RCT has compared a new technique to aid surgery for high-grade cataracts by disassembling the nucleus with a loop device inside the lens capsule prior to phacoemulsification [8]. The authors found that cumulative dispersed energy was 53\% higher in controls. Ultimately, scrutinising surgical outcomes in this way will contribute significantly to patient safety in its broadest definition.

\section{Compliance with ethical standards}

Conflict of interest The authors declare that they have no conflict of interest.

Publisher's note: Springer Nature remains neutral with regard to jurisdictional claims in published maps and institutional affiliations.

\section{References}

1. Keay L, Lindsley K, Tielsch J, Katz J, Schein O. Routine preoperative medical testing for cataract surgery. Cochrane Database Syst Rev. 2019;1:CD007293. https://doi.org/10.1002/14651858. CD007293.pub4

2. Cavallini GM, Saccarola P, D'Amico R, Gasparin A, Campi L. Impact of preoperative testing on ophthalmologic and systemic outcomes in cataract surgery. Eur J Ophthalmol. 2004;14:369-74.

3. Lira RP, Nascimento MA, Moreira-Filho DC, Kara-Jose N, Arieta CE. Are routine preoperative medical tests needed with cataract surgery? Pan Am J Public Health. 2001;10:13-7.

4. Schein OD, Katz J, Bass EB, Tielsch JM, Lubomski LH, Feldman MA, et al. The value of routine preoperative medical testing before cataract surgery. Study of Medical Testing for Cataract Surgery. New Engl J Med. 2000;342:168-75.

5. Day AC, Donachie PH, Sparrow JM, Johnston RL. The Royal College of Ophthalmologists' National Ophthalmology Database Study of Cataract Surgery: Report 1, Visual Outcomes and Complications. Eye. 2015;29:552-60.

6. Kim BZ, Patel DV, Sherwin T, McGhee CN. The Auckland Cataract Study: assessing preoperative risk stratification systems for phacoemulsification surgery in a teaching hospital. Am J Ophthalmol. 2016;171:145-50.

7. Agrawal V, Upadhyay J, Indian Cataract Risk Stratification Study Group. Validation of scoring system for preoperative stratification of intra-operative risks of complications during cataract surgery: Indian multi-centric study. Indian J Ophthalmol. 2009;57:213-5.

8. Ianchulev T, Chang DF, Koo E, MacDonald S, Calvo E, Tyson FT, et al. Microinterventional endocapsular nucleus disassembly: novel technique and results of first-in-human randomised controlled study. Br J Ophthalmol. 2019;103:176-80. 